\title{
Development of an Integrated Platform for Cross-Correlative Imaging of Biological Specimens in Liquid using Light and Electron Microscopies
}

\author{
D. Alphonse Fischer ${ }^{1,4}$, Daan Hein Alsem ${ }^{1}$, Benjamin Simon ${ }^{2}$, Tanya Prozorov ${ }^{3}$ and Norman Salmon ${ }^{1}$ \\ 1. Hummingbird Scientific, Lacey, WA, USA. \\ 2. The Evergreen State College, Olympia, WA, USA. \\ 3. Division of Materials Sciences and Engineering, Ames Laboratory, Ames, IA, USA. \\ 4. Currently at Department of Chemistry, University of Georgia, Athens, GA, USA.
}

One major obstacle to studying complex biological structures and their interactions with the environment is that no single microscopy technique can span all the length scales of interest. Therefore, correlative techniques [1-3] that combine the strengths of two or more imaging techniques, for example, light microscopy for rapid survey of the biological specimen and electron microscopy for providing information at the sub-cellular level, are being actively developed. Moreover, the vacuum requirements of an electron microscope often mean that complex sample preparation methods, such as cryogenic freezing or embedding the samples in resins [2], are needed and these techniques have been known to introduce artefacts $[4,5]$.

Here, we present early results from a prototype cross-correlative platform; developed at Hummingbird Scientific that allows us to translate from light microscopy (LM) to scanning electron microscopy (SEM) to transmission electron microscopy (TEM) while maintaining the samples in their native aqueous environment. The heart of the design is the detachable tip [6] from a Hummingbird Scientific liquid flow TEM holder and attachments that integrate the tip with standard LM and SEM equipment (see Figure 1 (a) to (d)). We can also maintain flow to the liquid cell while imaging in all three platforms.

In Figure 2, we present a series of LM, SEM, and STEM images taken from the same specimen (ironbinding protein solution) to demonstrate correlative capability of the system. The protein samples are patterned directly on the membrane windows using BioForce Nano eNabler molecular printer. The presence of the protein pattern was confirmed using LM, as shown in 2(a), before transfer to the electron microscopes. The SEM image in 2(b) shows the macrostructure of the printed dots, whereas the detailed form and size of individual iron-incubated protein micelles could be resolved using scanning transmission electron microscopy (STEM) imaging. To further enhance the sample locating capability, we had also patterned the electron transparent $\mathrm{SiN}$ membrane windows with features as shown in Figure 3. Here, the specimens are CHSE-214 (salmon embryo) that had been directly incubated on biofunctionalized chips and examined using LM and SEM. Lastly; we discuss the potential of integrating platform together with other light microscopy based techniques such as florescence microscopy.

\section{References:}

[1] Y Su et al, Biophys Rev 2 (2010) p. 121.

[2] K Cortese, A Disapro and C Tacchetti, J Histochem Cytochem 57 (2009), p. 1103.

[3] JM Robinson et al, J Histochem Cytochem 49 (2001), p. 803.

[4] A Al-Amoudi, D Studer and J Dubchet, J Struct Biol (2005) 150, p. 109.

[5] MV King, Cell Biophys (1991) 18, p. 31. 
[6] U.S Patent 8, 059, 271 and U.S. Patent 8, 102, 523.

[7] The authors, DAF, DHA and NS, acknowledge funding from NIH Grant \#1R43GM098132-01. The work at Ames Laboratory was supported by the U.S. Department of Energy, Basic Energy Sciences, Materials Sciences and Engineering Division. The Ames Laboratory is operated for the U.S. Department of Energy by Iowa State University under contract DE-AC02-07CH11358. The assistance of Dr. See Wee Chee is much appreciated.
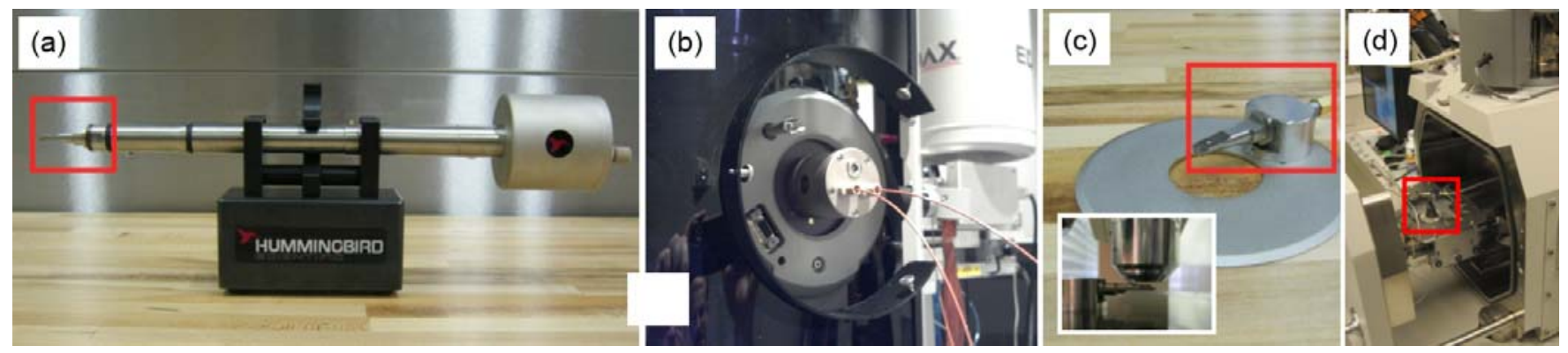

Figure 1. Cross-correlative platform for imaging biological specimens using light and electron microscopies. (a) Hummingbird Scientific liquid flow TEM holder in (b) a FEI Titan. (c) Attachment for standard upright light microscope (depicted in insert). (d) Attachment for SEM and (e) after loading into a FEI Quanta 3D SEM/FIB. The liquid flow tip highlighted in red boxes can be easily transferred between different systems and flow can be maintained in all platforms.

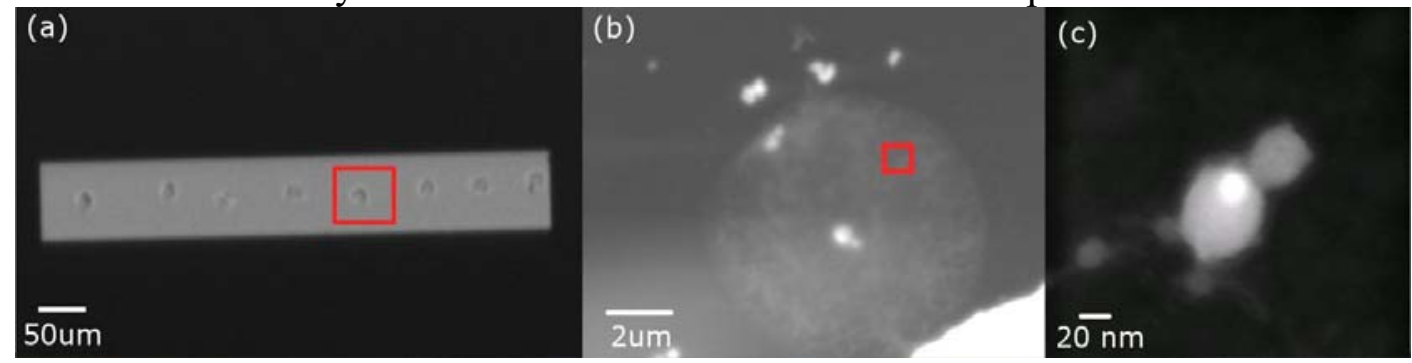

Figure 2. Correlative LM, SEM, and TEM images of iron-incubated protein micelles. In (a), the protein solution on the window membrane is visible in LM. In (b), the SEM shows the protein $\sim 10 \mu \mathrm{m}$ spot depicted in (a). In (c), the STEM image shows the detailed form and size of individual micelles. Red squares in (a) and (b) indicate the ROI.

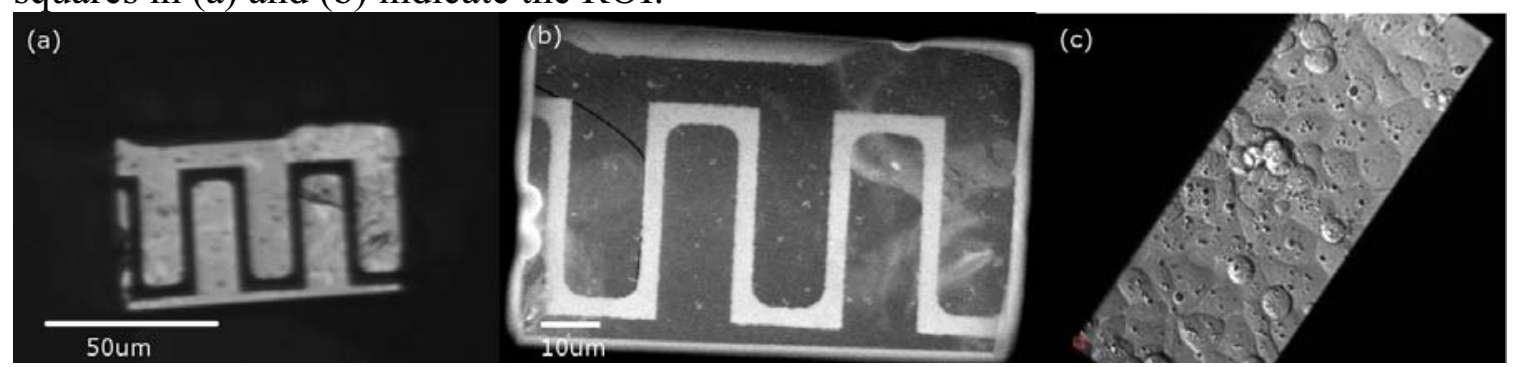

Figure 3. Correlated LM and SEM images of a sample cell containing CHSE-214 (salmon embryo) cells cultured directly on the sample window ((a) and (b), respectively). The method for culturing cells on the windows was later optimized to provide a confluent monolayer of cells across the window, as shown in the LM (differential interference contracts) image in (c). 